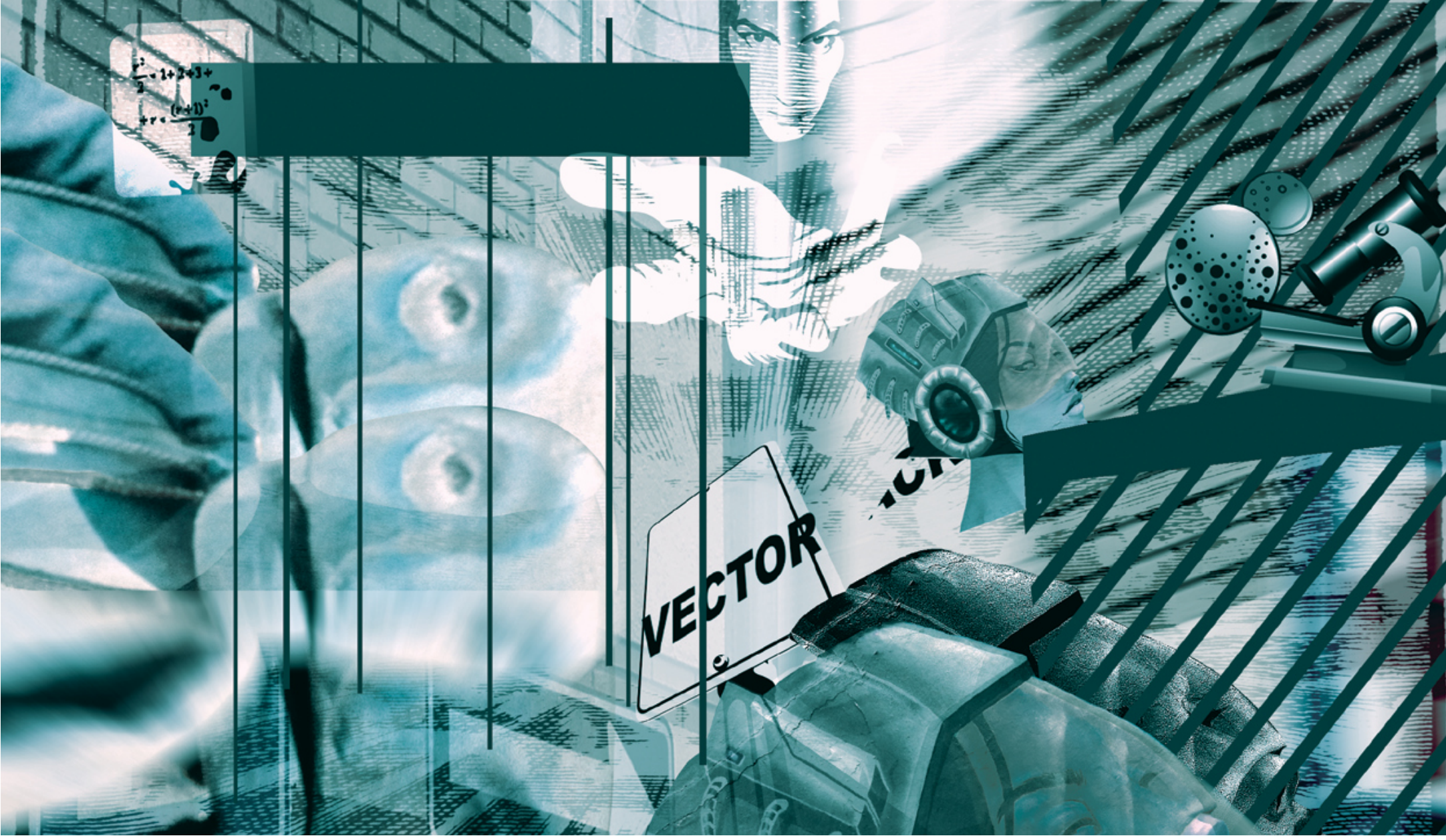

\title{
Saberes ancestrales y tradicionales vinculados a la práctica pedagógica desde un enfoque intercultural: un estudio realizado con profesores de ciencias en formación inicial
}

Ancestral and traditional knowledge linked to pedagogical practice from an intercultural approach: a study carried out with teachers of sciences in initial training

Conhecimentos ancestrais e tradicionais ligados à prática pedagógica a partir de uma abordagem intercultural: um estudo realizado com professores de ciências em formação inicial 


\section{Marisol Uribe Pérez ${ }^{1}$}

2. Candidata a Doctora en Educación, Doctorado Interinstitucional en Educación, Universidad Distrital (DIE-UD). Grupo de investigación DIDAQUIM. Línea de investigación cambio didáctico y formación del profesorado de ciencias. Directiva Docente: Secretaría de Educación Distrital (SED); correo electrónico: marisoluribeperez@ gmail.com

\section{DOI: https://doi.org/10.36737/01230425.v2.n37.2019.2148}

Fecha de recepción: 28 de enero de 2019 / Fecha de aprobación: 30 de agosto de 2019

\begin{abstract}
Resumen
El presente artículo expone los resultados de una investigación doctoral acerca de los saberes ancestrales y tradicionales vinculados a la práctica pedagógica, desde un enfoque intercultural, en profesores de ciencias en formación inicial. El enfoque metodológico es cualitativo e integra el estudio de caso colectivo; como técnica para recoger la información se consideró una entrevista colectiva semiestructurada. Los resultados abordan las estrategias empleadas por los profesores en las clases de ciencias, las cuales plantean la importancia de considerar la pluralidad de cosmovisiones y la descolonización del saber en la práctica pedagógica.
\end{abstract}

\section{Palabras clave:}

Saberes ancestrales y tradicionales, práctica pedagógica, profesores de ciencias en formación inicial, enfoque intercultural.

\section{Abstract}

This article presents the results of a doctoral research about ancestral and traditional knowledge linked to pedagogical practice from an intercultural approach, in science teachers in initial training. The methodological approach is qualitative and integrates the collective case study, as technique to collect the information, a semi-structured collective interview was considered. The results show the strategies used by teachers in science classes, which raise the importance of considering the plurality of worldviews and the decolonization of knowledge in pedagogical practice.

\section{Keywords:}

Ancestral and traditional knowledge, pedagogical practice, science teachers in initial training, intercultural approach.

\section{Resumo}

Este artigo apresenta os resultados de uma pesquisa de doutorado sobre conhecimentos ancestrais e tradicionais ligados à prática pedagógica a partir de uma abordagem intercultural, em professores de ciências em formação inicial. A abordagem metodológica é qualitativa e integra o estudo de caso coletivo, como técnica de coleta de informações, foi considerada uma entrevista coletiva semiestruturada. Os resultados abordam as estratégias empregadas pelos professores nas aulas de ciências, as quais levantam a importância de considerar a pluralidade de visões de mundo e a descolonização do conhecimento na prática pedagógica.

\section{Palavras-chave:}

Conhecimento ancestral e tradicional, prática pedagógica, professores de ciências em formação inicial, abordagem intercultural. 


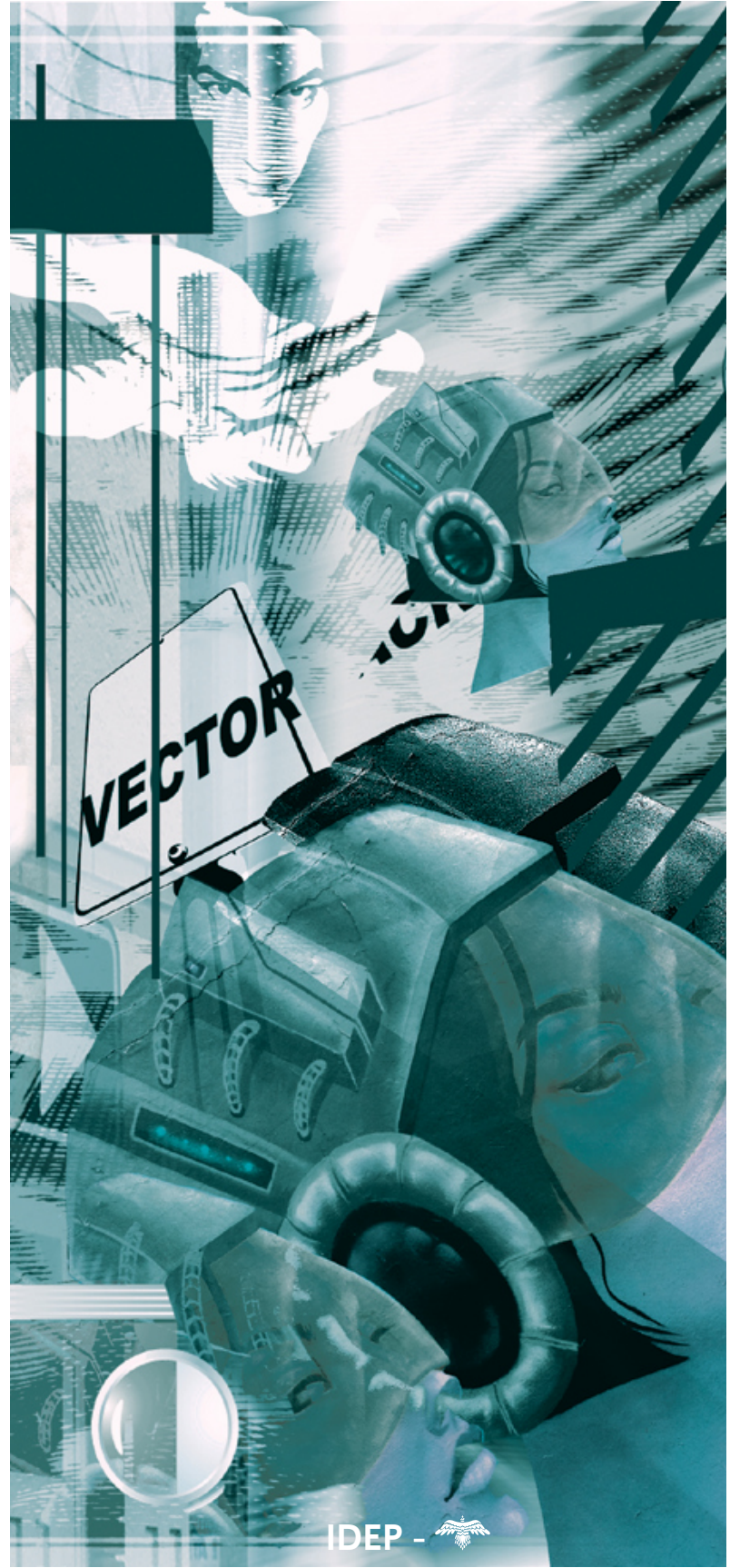

\section{Introducción}

$\mathrm{S}$ er maestros y maestras hoy, en sociedades pluriétnicas y multiculturales, implica asumir un enfoque intercultural en la educación. El enfoque intercultural es un paradigma aun emergente en la formación de profesores y en la enseñanza de las ciencias, a pesar de que la educación intercultural se viene considerando desde hace varias décadas. Este enfoque incorpora en la escuela el pluralismo epistemológico, así como la herencia cultural y ancestral de las comunidades.

La perspectiva intercultural expresa una crítica a la homogenización y a la aculturación de los pueblos originarios y comunidades afrodescendientes, al cientificismo y a la violencia epistémica. Plantea la posibilidad de una descolonización en aspectos de orden epistémico, filosófico y pedagógico; ya que la escuela por siglos ha privilegiado el saber occidental, debido al legado de la modernidad y a la colonización cultural y educativa.

El enfoque intercultural en la formación de profesores, y en la enseñanza de las ciencias, involucra reconocer y re-vivir nuestra ancestralidad, otros saberes y otros significados, reafirmando diferentes discursos e identidades. La universidad está llamada a contribuir a la construcción de una sociedad y ciudadanía intercultural, donde se conjugue lo occidental y lo no occidental, cosmogonías y lenguajes diferentes, el buen vivir "Sumak Kawsay", lo ontológico, lo espiritual, lo humano, lo colectivo y lo ético. Todo ello, dando lugar a la alteridad y a la reciprocidad. "Es urgente el diálogo con otras educaciones pero desde una perspectiva pedagógica intercultural, crítica y (de) colonizada" (Gómez, Galindo, García y González, 2014, p. 160). El diálogo intercultural es una opción contra el cientificismo, el cual ha tenido una trayectoria histórica en la enseñanza de las ciencias (Santos, 2014).

En Colombia, el Ministerio de Educación Nacional sugiere, en el documento Sistema Colombiano de Formación de Educadores y Lineamientos de Política (MEN, 2013), la diversidad y la interculturalidad como principios fundamentales para los programas de licenciatura. En este sentido, existe un gran desafío en cuanto a la transición de currículos monoculturales hacia currículos interculturales en la formación de profesores. 
Respecto a investigaciones sobre la educación intercultural, Aguado y Malik (2009) indican que es preciso adoptar un enfoque intercultural en la formación docente y en la práctica escolar, propiciando un escenario de intercambio en la formación inicial y en servicio del profesorado. En Colombia, la línea de investigación "Contexto, diversidad cultural y enseñanza de las ciencias", del Doctorado Interinstitucional en Educación, Universidad Distrital, ha venido desarrollando diferentes investigaciones que abordan las prácticas de los profesores en relación a la diversidad cultural y la interculturalidad. Algunas de ellas son: "Concepciones de los profesores y los ámbitos de sus prácticas, dos estudios de caso" (Molina y Utges, 2011); "La enseñanza como puente entre conocimientos científicos escolares y conocimientos ecológicos tradicionales" (Molina y Mojica, 2013); y Concepciones de los profesores acerca del fenómeno de diversidad cultural y sus implicaciones en la enseñanza de las ciencias (Molina, Mosquera, Utges, Mojica, Cifuentes, Reyes y Pedreros, 2014).

Al respecto, también vale la pena destacar otras investigaciones, como "Creencias sobre la educación intercultural y prácticas de enseñanza de profesores de secundaria de la población indígena Yaqui en México" (Fernández, Torres y García, 2016); o la de Leiva (2010), quien establece algunas reflexiones sobre la práctica educativa y la interculturalidad desde la perspectiva docente, considerando la diversidad cultural como referente fundamental de las prácticas educativas. Al tiempo, investigaciones como las de Leeman y Ledyoux (2003), indican que los debates en educación intercultural se encuentran alejados de las prácticas de los profesores en el aula de clase.
Teniendo en cuenta lo anterior, el presente artículo se pregunta por cuáles son los saberes ancestrales y tradicionales vinculados a las prácticas pedagógicas de los profesores de ciencias en formación inicial, en relación al enfoque intercultural en la enseñanza de las ciencias. La práctica pedagógica se concibe como un espacio donde el profesor pone en evidencia su saber pedagógico y otro tipo de saberes (disciplinares/científicos, cotidianos, tradicionales, didácticos), es un lugar donde se intercambian y apropian diversos discursos, con sus respectivos significados.

Respecto a la práctica de los profesores en formación inicial, existen diversas denominaciones y conceptualizaciones: práctica docente, práctica pedagógica, práctica educativa y práctica pedagógica investigativa. La práctica de los profesores se ubica en diferentes paradigmasomodelos(técnicos, humanistas,investigativos),loscuales determinan el enfoque de la acción pedagógica (Rodríguez, 2002).

De acuerdo a Molina, et al. (2014), son numerosas las investigaciones que muestran una relación estrecha entre las concepciones de los profesores y sus propuestas de enseñanza. Así mismo, Yuen (2009) plantea que la competencia y la sensibilidad intercultural de los profesores se encuentran estrechamente vinculadas con una enseñanzaadecuaday pertinente en contextos de diversidad cultural.

Esta investigación se suscribe en el énfasis de Educación en Ciencias, del Doctorado Interinstitucional en Educación de la Universidad Distrital (DIE-UD). Los resultados contribuyen a explicar por qué es importante considerar el enfoque intercultural en la práctica pedagógica, integrando los saberes ancestrales y tradicionales como contenidos de referencia para la construcción de conocimiento en las ciencias de la naturaleza.

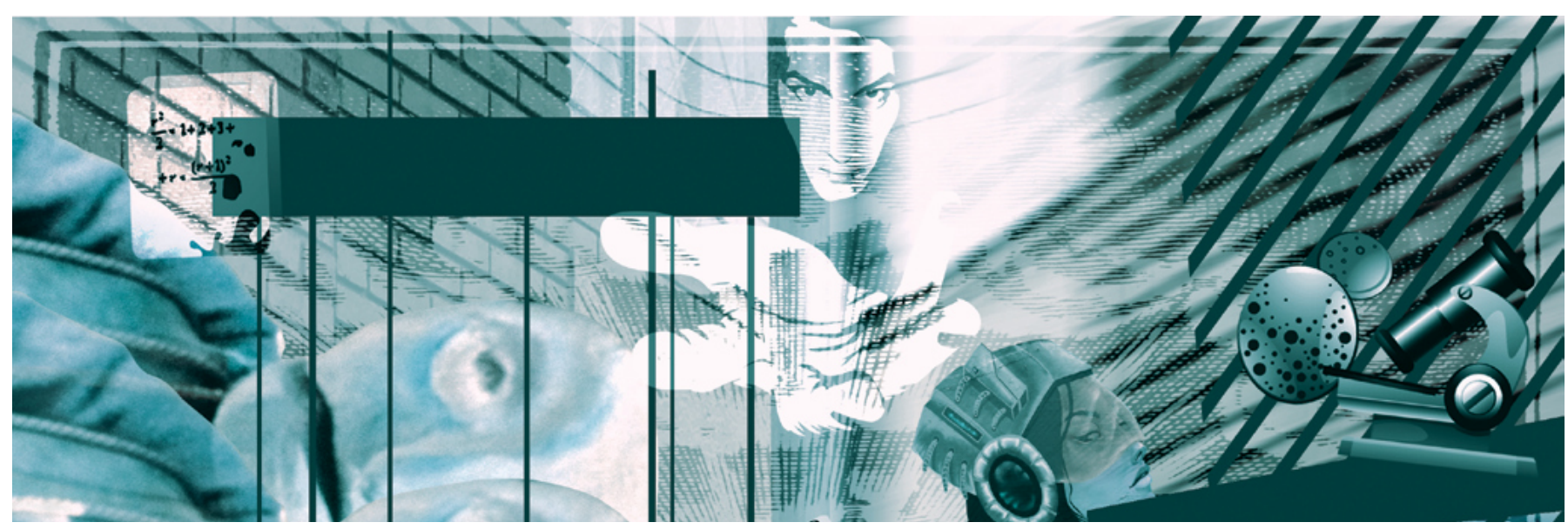

60 EduCACIÓn y CIUdAd No. 37 JULIO - DICIEMBRE DE 2019 ISSN 0123-425 WeB-ONLINE 2357-6286 PP. 57-71 


\section{Metodología}

Tabla 1. Diseño Metodológico

\section{Enfoque}

Cualitativo y emergente

\section{Estrategia}

Estudio de Caso Colectivo (Stake, 1999; Creswell, 2005)

Nueve programas de licenciatura en ciencias naturales
Instrumentos

\section{Participantes}

Entrevista colectiva 45 profesores de ciencias en formación inicial: Indígenas, semiestructurada afrodescendientes y mestizos

Tabla 2. Fases de la investigación

Exploración y diseño

Aplicación

Sistematización

Construcción del contexto conceptual y diseño metodológico

Validación de instrumento

Selección de los participantes

Aplicación de instrumento

Transcripción de entrevistas

Triangulación de instrumentos usando el programa informático Atlas ti

Triangulación teórica para la interpretación de las prácticas pedagógicas, teniendo en cuenta los fragmentos de contenido con sentido y significado para la investigación

Conclusiones y reflexiones finales

Tabla 3. Casos analizados en el estudio de caso colectivo

Licenciatura en educación básica con énfasis en ciencias naturales y educación ambiental

Universidad del Valle, sede Pacífico

Licenciatura en educación básica con énfasis en ciencias naturales y educación ambiental Universidad del Valle, sede Pacífico

3 Licenciatura en educación básica con énfasis en ciencias naturales y educación ambiental Universidad de Antioquia

Licenciatura en biología y química

Universidad del Atlántico

5

Licenciatura en biología y química

Universidad de Caldas

Licenciatura en educación básica con énfasis en ciencias naturales y educación ambiental

Universidad del Cauca

Licenciatura en ciencias naturales: Física, química y biología

Universidad Surcolombiana

Licenciatura en ciencias naturales y educación ambiental

Universidad Popular del Cesar

9

Licenciatura en química

Universidad Distrital 


\section{Resultados}

\section{Gráfico 1. Saberes ancestrales y tradicionales vinculados a la práctica docente}

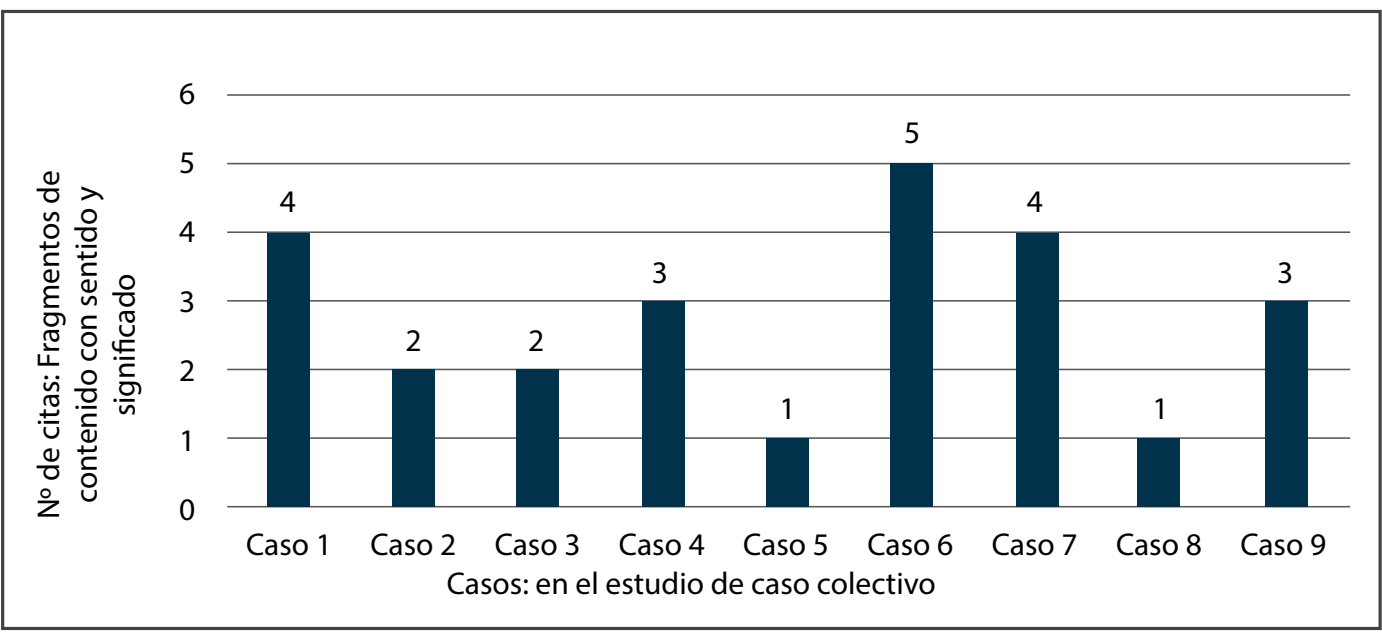

Los saberes ancestrales o tradicionales, vinculados en la práctica docente de los profesores de ciencias en formación inicial, contemplan los siguientes aspectos.

\section{Cosmovisiones, valores y prácticas de las comunidades indígenas}

La cosmovisión y prácticas indígenas, como la asamblea y el círculo de la palabra, son incluidas en la práctica pedagógica con la integración de una pedagogía intercultural:

Consideramos valores y prácticas de las comunidades indígenas como la asamblea para hacer conversatorios en la maloca. La maloca es el salón de clase, es un espacio donde se construye conocimiento, integramos una tradición ancestral que es el círculo de la palabra, nos organizamos en mesa redonda para que todos interactúen y puedan compartir sus saberes. También se fomentan valores ancestrales (PCFI, No. 1. Licenciatura en educación básica con énfasis en ciencias naturales y educación ambiental, Universidad del Cauca).

De acuerdo a Peña (2014), es necesario reconocer una episteme tradicional y analizar la pluralidad de saberes en la tradición del conocimiento, en los escenarios socioculturales que se producen, para no seguir perpetuando las formas de reproducción social, cultural y educativa bajo una visión unilateral respecto al conocimiento.

\section{Saberes campesinos}

Los saberes campesinos implican una serie de experiencias y prácticas tradicionales:

Cuando empezamos a indagar acerca de la función de relación en las plantas y los tropismos, pensé en el campo. Le expliqué a mi mamá en qué consistía el fototropismo, entonces mi mamá me dijo: Eso es lo que pasa cuando uno siembra café, se tiene que sembrar cuando está haciendo más sol, porque así la plántula germina bien. Cuando hay mucho invierno la plántula no sale, sino que tiene un desvío, porque la zona está húmeda, entonces se queda allí y se demora más tiempo en salir. Los niños que vienen del campo saben lo que pasa con las plantas, ellos no hablan en términos científicos, pero sí saben muy bien que es el fototropismo o el hidrotropismo (PCFI, No. 3, Licenciatura en educación básica con énfasis en ciencias naturales y educación ambiental, Universidad del Cauca, Entrevista colectiva).

Existe un conocimiento campesino que plantea otra epistemología (Schmelkes, 2004), la cual implica un saber a través de prácticas empíricas en el campo. Como lo plantea la profesora de ciencias, es importante cuestionar que el único conocimiento válido es el 
científico; los campesinos, así como los indígenas, representan otra tradición, otra racionalidad. El saber campesino es legítimo en la medida en que históricamente ha podido demostrar su viabilidad y utilidad.

\section{Patrimonio biocultural}

En la enseñanza, el patrimonio biocultural vincula los saberes culturales más allá de la visita a museos y colecciones que conservan dicho patrimonio. Significa empezar a pensar cómo la diversidad y expresiones culturales empiezan a ser integradas en la educación. Uno de los proyectos pedagógicos investigativos retoma el patrimonio cultural, asociado a una zona de reserva natural campesina en Santa Rosa, Cauca.

En nuestro proyecto pedagógico investigativo en torno a la conservación, tenemos en cuenta los relatos de los abuelos, sus historias, porque ellos cuentan cómo era Santa Rosa antes, ellos narran sus conocimientos y experiencias, lo que saben acerca de la biodiversidad de esta zona. Allí encontramos también un patrimonio cultural, valores, conocimientos y formas de relacionamiento con la naturaleza que, a través de la práctica pedagógica, integramos con entrevistas e interacción con la comunidad que ha habitado este territorio por mucho tiempo (PCFI, No. 5, Licenciatura en educación básica con énfasis en ciencias naturales y educación ambiental, Universidad del Cauca, entrevista colectiva).

En su práctica, la profesora da un valor fundamental a los saberes de las personas que han habitado por décadas el territorio donde se encuentra la reserva; allí crean espacios de diálogo escuelacomunidad, que permiten promover el intercambio de experiencias y conocer los saberes tradicionales que hacen parte del patrimonio cultural campesino. En la enseñanza, este patrimonio inmaterial implica un proceso pedagógico centrado en conocimientos y valores que subyacen a un pueblo o sociedad, donde los adultos mayores son poseedores del saber ancestral (Boen, Piñuel, Zubillaga, Dalzotto, y Sharry, 2017).

\section{Papel de la mujer afrodescendiente}

Las mujeres afrocolombianas tienen un papel fundamental dentro de la sociedad afrodescendiente: muchas de ellas han demostrado su lucha a favor de su cultura desde la época de la Colonia. La mujer afro estuvo marginada por mucho tiempo de la educación, la política, la toma de decisiones y la ciencia; no obstante, un amplio número de ellas se ha emancipado a través de la historia, sobresaliendo en diferentes aspectos.
Mi trabajo de grado, que se trabaja desde la práctica pedagógica, es sobre las concepciones de docentes y estudiantes acerca de la mujer afro y la ciencia. Tuve un acercamiento con los estudiantes de la institución y era cómo ellos ven a la mujer afro desde su cultura y cómo los docentes también ven a la mujer afro. La mujer afro, en cierta manera, conserva una serie de tradiciones, ha sido símbolo de libertad y de lucha (PCFI, No. 2, Licenciatura en educación básica con énfasis en ciencias naturales y educación ambiental, Universidad del Valle, sede Pacífico, entrevista colectiva).

Existe un saber ancestral y tradicional perpetuado por las mujeres sabedoras afro del Pacífico colombiano, en temas como cultura culinaria, biodiversidad, plantas medicinales, bebidas tradicionales y partería. El saber y la voz de la mujer afro también se evidencian en la literatura, la música, la danza y las artesanías, contribuyendo a recuperar la cultura a través de su trabajo.

\section{Planes de estudios etnoeducativos}

De acuerdo al Ministerio de Educación Nacional (1994), el objetivo de la etnoeducación es el reconocimiento de la diversidad cultural en todas las escuelas y colegios del país. La etnoeducación permite el fortalecimiento de los planes de estudio:

En el trabajo de grado, como parte de la práctica, he abordado la etnoeducación para el fortalecimiento de los planes de estudio de grado quinto, integrando conocimientos ancestrales que forman parte de la comunidad afrocolombiana. Realicé una entrevista a una partera del sector de la Bocana, también entrevisté a dos expertos sobre el tema y busqué opiniones de los sabedores de Buenaventura. Con los niños resalté la importancia de los saberes ancestrales de la Región Pacífica (PCFI, No. 3, Licenciatura en educación básica con énfasis en ciencias naturales y educación ambiental, Universidad del Valle, sede Pacífico, entrevista colectiva).

Este es un ejemplo de cómo diseñar un plan de estudios etnoeducativo en contexto. La integración de los saberes afrodescendientes e indígenas en el currículo permite pensar la escuela desde una perspectiva decolonial, basada en la pluralidad de saberes. Esto contribuye al desprendimiento de la dominación colonial en el ámbito educativo. 


\section{Conceptos que permiten establecer el diálogo de saberes}

Existen conceptos como la menstruación, que implican significados y prácticas culturales diversas.

En mi práctica y trabajo de grado he estudiado los saberes ancestrales sobre la menstruación aquí en la comunidad afro del Pacífico, para ver las creencias, las prácticas desarrolladas por las mujeres afrodescendientes y los cuidados durante el periodo de la menstruación, todo esto también hace parte de nuestro patrimonio cultural, de la misma medicina tradicional. Sabemos que existen muchas ideas en cada cultura sobre la menstruación (PCFI, No. 5, Licenciatura en educación básica con énfasis en ciencias naturales y educación ambiental, Universidad del Valle, sede Pacífico, entrevista colectiva).

Los conceptos que se enseñan en ciencias son polisémicos, dependiendo de cada contexto histórico y cultural. La pluralidad de significados refleja las cosmovisiones de cada comunidad. El perfil conceptual de una idea considera que los diversos puntos de vista sobre la realidad están asociados a contextos específicos que le son apropiados, por lo que no se debe privilegiar una forma de pensar sobre otra (Mortimer y El Hani, 2014).

\section{Prácticas agrícolas ancestrales}

Algunos saberes tradicionales se ponen en evidencia a través de las prácticas agrícolas.

Cuando estudié materia, energía y electromagnetismo nos explicaban los efectos de las cargas, leí un artículo donde se mencionaba cómo las cargas y el electromagnetismo de la tierra generan un impacto en los cultivos. Esas cargas actúan de alguna manera en el crecimiento de las plantas y los campesinos utilizan esto y las fases lunares para sembrar. Yo tuve en cuenta estos aspectos en clase (PCFI, No. 1, Licenciatura en ciencias naturales: Física, química y biología, Universidad Surcolombiana, entrevista colectiva).

Las propiedades eléctricas y los campos magnéticos que rodean a la Tierra responden a un proceso de ajuste de las cargas eléctricas de nuestro planeta con respecto al campo eléctrico del Sol. El estudio de los campos eléctricos y sus efectos sobre los organismos se ha llevado a cabo desde hace mucho tiempo. Las repuestas de los diferentes organismos a los campos eléctricos y magnéticos dependen del organismo y su estructura, del medio de crecimiento en el cual se encuentre, de la fuerza del campo, el tiempo de exposición, la forma y frecuencia del campo, entre otros factores (Ohshima, Tamura y Sato, 2007).

\section{Medicina tradicional y plantas medicinales}

La medicina tradicional y el estudio de plantas medicinales son elementos incorporados a través de la fitoquímica en la enseñanza de las ciencias.

A mí me parece interesante encontrar por qué sirve, o por qué no, cierta planta; a raíz de eso pienso que la medicina tradicional es una manera de tener en cuenta los saberes tradicionales. Esto lo he ido apropiando en el aula de clase, trabajando algunas cosas que he adelantado en mi tesis, para ir de ese conocimiento tradicional a un conocimiento científico. Mi tesis se llama "Análisis fitoquímico preliminar de la especie vegetal Begonia erythrofhilla y su evaluación contra la nefropatía diabética", mi padre, de manera tradicional, la toma para problemas renales. A partir de ahí se planteó hacer un tamizaje fitoquímico para encontrar su agente activo (PCFI, No. 4, Licenciatura en ciencias naturales: Física, química y biología, Universidad Surcolombiana, entrevista Colectiva).

Para este profesor resulta importante estudiar la medicina tradicional, relacionando la etnobotánica y la composición química de las plantas. Su interés se centra en encontrar los principales metabolitos secundarios que pueden estar asociados a los usos terapéuticos que se le atribuyen a una especie de planta usada por su papá, para el tratamiento de una enfermedad renal, a través de la medicina tradicional.

El reconocimiento fitoquímico de algunas plantas usadas en la medicina tradicional es un elemento que vincula los saberes ancestrales y el conocimiento científico. La relación de los metabolitos primarios y secundarios con el uso tradicional de algunas plantas es un tema ampliamente estudiado; Scarpetta (2017) presenta un estudio acerca de la relación entre los metabolitos secundarios, o alcaloides en la planta de Erythroxylum Coca var (Coca), y su uso medicinal en la población indígena Nasa.

\section{Método científico y saberes tradicionales}

Poner en relación el método científico con los saberes tradicionales, y con algunas problemáticas del contexto, se planteó de la siguiente manera:

Los estudiantes pueden mezclar lo tradicional con el método científico. Para tratar esto, se partió de la observación de una problemática del barrio. Se les recomendó observar diferentes tipos de contaminación ambiental. Los estudiantes debían generar preguntas sobre la problemática e hipótesis teniendo en cuenta sus conocimientos. Unos estudiantes mencionaron que veían peces muertos y decían que los peces mueren de hambre porque, como el río estaba contaminado y el agua sucia, el pez no podía observar el alimento. Los estudiantes formularon la 
pregunta ipor qué mueren los peces en ese hábitat?, llegaron a la hipótesis de que murió por hambre y porque no pudo cazar su alimento. Luego se hizo la experimentación, esta consistía en preguntar acerca de cómo se explica ese fenómeno a la familia o personas del barrio, también se podía buscar por Internet. Se sacaron conclusiones a nivel grupal e individual. Por último, los estudiantes tenían el deber de socializar sus conclusiones y recomendaciones para mitigar la problemática con la comunidad (PCFI, No. 5, Licenciatura en ciencias naturales: Física, química y biología, Universidad Surcolombiana, entrevista colectiva).

En el marco de una visión eurocéntrica y positivista el método científico es un tema obligado de la educación en ciencias; por tanto, buscar su relación con los saberes tradicionales y los elementos del contexto resulta interesante para lograr el diálogo de saberes y nuevas formas de circulación entre conocimientos.

\section{Problemáticas ambientales}

Los saberes ancestrales y tradicionales han sido integrados a la práctica docente mediante el análisis del impacto de una problemática ambiental en comunidades locales:

En el trabajo de grado y en la práctica trabajamos macro invertebrados como indicadores del suelo, trabajamos en el municipio de Chiriguaná porque queda cerca de la mina de carbón Drummond, para ver cómo la mina está afectando al suelo y cómo los macro invertebrados que vemos por ahí (cucarachas, ciempiés, lombrices, larvas, hormigas), ayudan a identificar la calidad del suelo. Le preguntamos a unos señores acerca del tema, ellos nos hacían comentarios, por ejemplo, que cuando va a llover se ven muchas hormigas en el suelo hacia una dirección, si la tierra está de algún color dicen que está ocurriendo algo en el suelo por la contaminación, porque antes el uso del suelo era diferente, habían ciertos animales y plantas que ya no hay. Cuando analizamos el suelo vimos que el $\mathrm{pH}$ es muy ácido, entonces algunos animales no pueden estar. Los macro invertebrados participan en los procesos de humificación del suelo y en la descomposición de materia orgánica, la disminución del $\mathrm{pH}$ del suelo y la baja presencia de macroinvertebrados se debe a lo que está ocurriendo en las minas. Nosotros vimos que esos conocimientos tradicionales tienen una lógica (PCFI, No. 1, Licenciatura en química y biología, Universidad del Atlántico, entrevista colectiva).

Aquí se plantea una forma en que los saberes tradicionales, al igual que los conocimientos científicos, pueden explicar el impacto ambiental de la mina de carbón, de la empresa Estaunidense Drummond, sobre la calidad de los suelos y la presencia de macro invertebrados. Esta entidad privada de explotación, exportación y procesamiento de carbón, es la segunda empresa más importante de este tipo en el país, después del Cerrejón, que ha tenido algunos episodios polémicos también susceptibles de ser analizados en la escuela.

\section{Estudio de plantas nativas}

Se referenció una práctica de capilaridad con la Coccoloba uvifera, conocida popularmente como Uva de Playa, Uvero de Playa o Uvita Playera, planta nativa de la región.

Yo he realizado prácticas de capilaridad con mis estudiantes usando la tinta de la Uva Playera, que da un color morado. Esta fruta también sirve para elaborar mermelada y bebidas alcohólicas, esto es algo que culturalmente uno ya sabe y, si se tienen los recursos naturales ahí disponibles, porque existen en la institución, se pueden aprovechar para el aprendizaje (PCFI, No. 2, Licenciatura en química y biología, Universidad del Atlántico, entrevista colectiva).

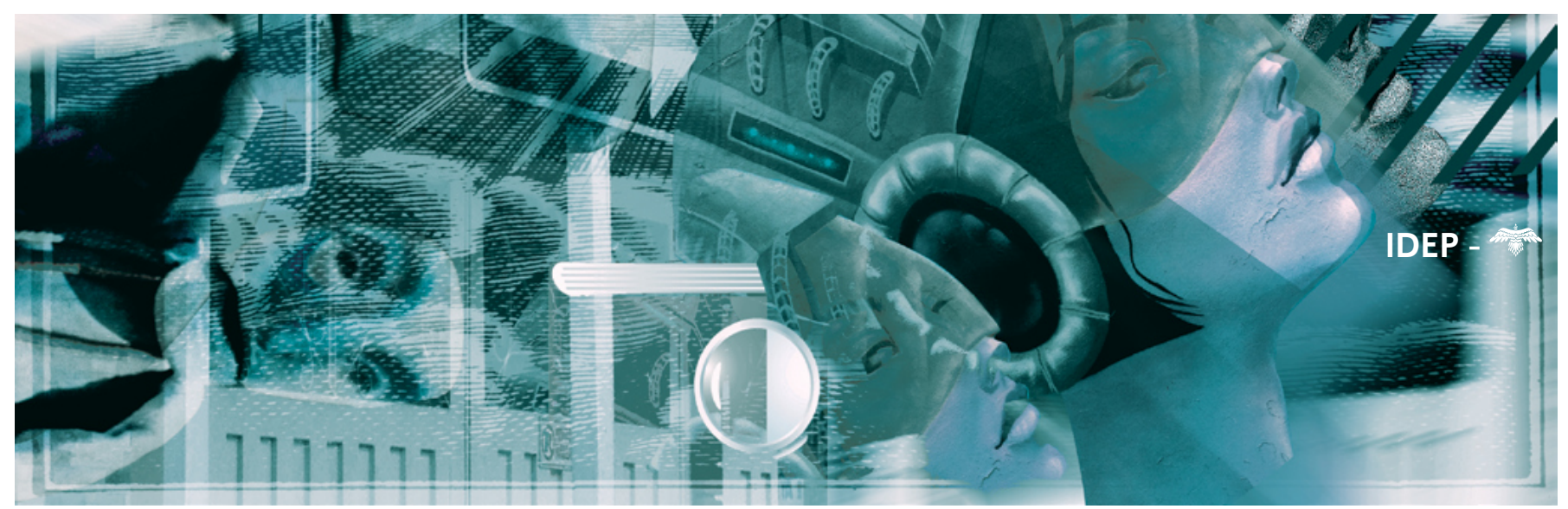

SABERES ANCESTRALES Y TRADICIONALES VINCULADOS A LA PRÁCTICA PEDAGÓGICA DESDE UN ENFOQUE INTERCULTURAL: UN ESTUdio REALIZADO CON PROFESORES DE CIENCIAS EN FORMACIÓN INICIAL / MARISOL URIBE PÉREZ 
En el ejemplo, la enseñanza de las ciencias considera el contexto y los saberes tradicionales acerca de las plantas nativas de una región. La Uva de Playa es una planta nativa de la Región Caribe que presenta múltiples aplicaciones susceptibles de ser estudiadas en clase. De acuerdo a Francis y Lowe (2000) sus usos son muy variados: Combustible; alimento; estimulante, a través del consumo de bebidas alcohólicas (vinos); fuente en la elaboración de tintes naturales y curtientes para el teñido de pieles; es útil además en la elaboración de artículos de madera y para la apicultura, sin contar con sus propiedades medicinales.

\section{Curiosidad de los estudiantes acerca de los saberes y prácticas de las comunidades indígenas}

Una profesora en formación Wayuu plantea que los saberes ancestrales no se encuentran incorporados al currículo de ciencias, razón que limita su implementación en la enseñanza. Por tanto, a pesar de ser conocedora de la sabiduría ancestral, solo comparte el saber de su comunidad cuando la curiosidad de los estudiantes lo permite:

Mis estudiantes saben que yo soy wayuu, a veces les hablo de mi comunidad, de mi lengua, cuando ellos preguntan. Pero eso se da, no porque yo lo programe para mis clases, es algo más espontáneo, que se da cuando hablamos de la parte ambiental o en alguna actividad, como la del Día de la Raza. Yo pienso que una forma de rescatar la cultura wayuu o cualquier cultura indígena es a través de la apropiación y reconocimiento de los saberes ancestrales, por eso tendría que haber reformas curriculares para que en los colegios y también universidades se tengan en cuenta; así, uno como profesor, en su práctica, tendría la posibilidad de integrarlos (PCFI, No. 5, Licenciatura en química y biología, Universidad del Atlántico, entrevista colectiva).

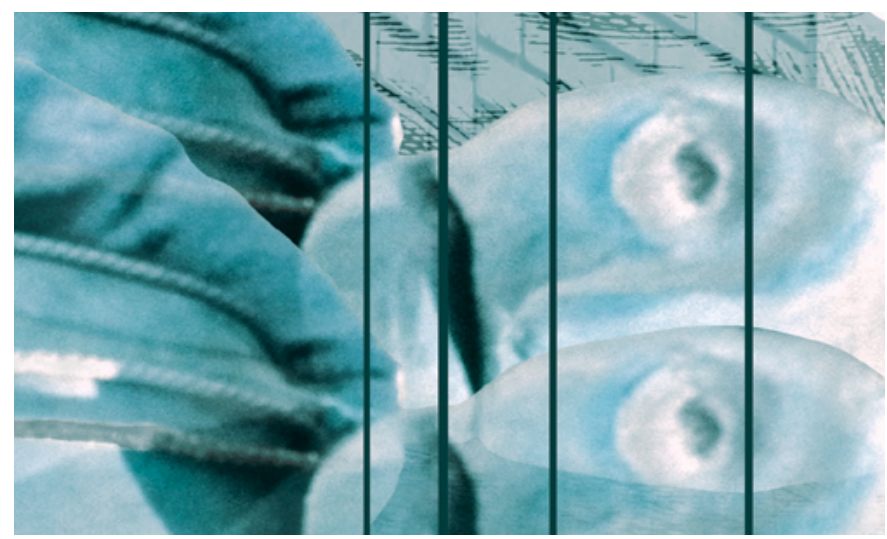

Lo anterior reafirma la necesidad de pasar de currículos monoculturales a currículos interculturales, al tiempo que la importancia de rescatar la sabiduría ancestral de los pueblos originarios por medio de una enseñanza intercultural de las ciencias. Por tanto, las iniciativas, orientadas a una enseñanza de las ciencias con enfoque intercultural, presentan nuevos retos en relación a la formación del profesorado.

\section{Historia de las ciencias}

Respecto a la historia de la química y su relación con los saberes tradicionales, se plantea que:

En las clases de química se debe tomar elementos de la historia para que los estudiantes entiendan la evolución de un concepto. Los saberes ancestrales son realmente importantes para saber la base de la ciencia actual, hacen parte de algunas culturas y de su historia. En mi labor docente he vinculado este tipo de conocimientos (PCFI, No.1, Licenciatura en química, Universidad Distrital, entrevista colectiva).

La sabiduría ancestral y tradicional ha nutrido el conocimiento científico, por lo que de ninguna manera es descabellada la idea de construir puentes entre la ciencia y otro tipo de saberes, llegando hasta la enseñanza de las ciencias. Una nueva corriente, denominada "ciencia posnormal", concepto propuesto y desarrollado por Funtowicz y Ravetz (1993), propone juntar la sabiduría ancestral con los conocimientos científicos de los expertos. La ciencia posnormal promueve la democratización científica en respuesta a las tendencias actuales de la posmodernidad.

\section{Patrimonio cultural}

La inclusión del patrimonio cultural, y de su relación con el conocimiento científico, es una posibilidad para asumir los desafíos de una educación científica intercultural. La ciencia y la enseñanza de las ciencias deben responder a los reclamos de aquellos pueblos que han visto históricamente suprimidos sus saberes.

En la enseñanza de reacciones químicas, desde la minería precolombina de la cultura de los Quimbaya, se incorporó los saberes ancestrales de esta comunidad, artífices de la minería. En la unidad didáctica se tuvo en cuenta el patrimonio cultural de este pueblo indígena (PCFI, No. 3, Licenciatura en química, Universidad Distrital, entrevista colectiva)

La minería y orfebrería quimbayas aportam conocimientos relevantes al campo de las ciencias. Desafortunadamente, el legado orfebre y las piezas de oro quimbayas fueron saqueadas durante la Colonia y regaladas por el gobierno colombiano a la corona 
española a finales del siglo XIX; hoy en día Colombia lucha por su repatriación. Los quimbayas plasmaron su cultura y cosmovisión en los objetos de oro, poniendo en evidencia sus avances en minería, orfebrería y avanzadas técnicas metalúrgicas.

\section{Conceptos estructurantes de las ciencias}

Algunos conceptos estructurantes de las ciencias son presentados como ejemplos para relacionar el saber tradicional con el conocimiento científico. En el caso de la biología, una noción fundamental es la evolución e incluye dos visiones de mundo muy importantes: la ciencia y la religión.

Yo he integrado los saberes ancestrales en la clase de biología en el tema de evolución, teniendo en cuenta lo que saben los estudiantes y su familia. Se analizó la relación entre la ciencia y la religión, examinando con los estudiantes la teoría de Darwin y el creacionismo, ya que muchos son católicos o cristianos. Se realizaron debates partiendo de algunas preguntas y videos (PCFI, No. 4, Licenciatura en química, Universidad Distrital, entrevista colectiva).

El Darwinismo presenta una visión de la naturaleza en el contexto científico y llegó a establecerse totalmente en el ámbito académico y, por otra parte, está la cosmovisión creacionista; para algunos ambas cosmovisiones son completamente diferentes, para otros, la religión ofrece algunos elementos que refuerzan lo planteado por la ciencia. La espiritualidad indígena presenta una serie de proposiciones que experimentaron un proceso de hibridación, mestizaje o sincretismo con la colonización.

Al respecto, Cajigas (2017) trata asuntos relacionados con las ontoepistemologías indígenas, indicando que, así como ha existido una violencia epistémica, también existe una violencia ontológica, por ello alerta sobre la necesidad de un diálogo intercultural y de posibilitar una ontología relacional; así, resulta fundamental, desde una dinámica de la relación, poner en diálogo el conocimiento occidental con la sabiduría y espiritualidad de los pueblos indígenas.

\section{Expresiones culturales como el Festival Petronio Álvarez}

El estudio también trabajó alrededor de la recuperación de saberes tradicionales a través de expresiones culturales, como el Festival Petronio Álvarez.

En el Colegio hemos propuesto un "Petronsito", un festival que emula al Festival Petronio Álvarez. Este festival de música del Pacífico reúne la gastronomía, las bebidas tradicionales, las artesanías y el vestuario afrodescendiente, permite recuperar nuestras tradiciones con los estudiantes (PCFI, No. 5, Licenciatura en educación básica con énfasis en ciencias naturales y educación, Universidad del Valle, sede Cali, entrevista colectiva).
El festival escolar retoma los saberes y expresiones de la comunidad afrodescendiente del Pacífico colombiano; un homenaje a la tradición oral que mantiene la memoria viva de su música, danza, literatura, cultura gastronómica, bebidas tradicionales, medicina tradicional, artesanías y vestuario. El festival integra diferentes voces, sonidos, olores, colores y sabores; ritmos de la región son interpretados a través de instrumentos tradicionales; allí se organizan diferentes conversatorios, presentaciones artísticas, muestras y exhibiciones. La iniciativa ha sido rescatada por Sevilla y Domingo (2017), quienes exponen su importancia durante los últimos por 21 años, así como su impacto en el conocimiento, apropiación y valoración del patrimonio cultural inmaterial de la región del Pacífico colombiano.

\section{Huerta escolar}

A través del trabajo en la huerta escolar se pueden compartir saberes tradicionales sobre las plantas:

Propusimos una huerta que denominamos "Enraízate" y ahí trabajamos la interculturalidad teniendo en cuenta saberes acerca de ciertas plantas. También trabajamos el tema de la adopción igualitaria. En la huerta escolar sembramos con los estudiantes plantas aromáticas y medicinales. Los niños seleccionaron una planta que conocían y que querían cuidar, la temática era: Adopta tu planta y cuídala (PCFI, No. 1, Licenciatura en educación básica con énfasis en ciencias naturales y educación ambiental, Universidad de Antioquia, entrevista colectiva).

La huerta escolar es una posibilidad de enseñar las ciencias desde una perspectiva intercultural y resignificar los saberes ancestrales y tradicionales en la escuela. En ella se pueden fusionar los conocimientos escolares y los saberes que han recibido los estudiantes de sus padres y abuelos. Particularmente, las personas que han vivido en el campo transmiten a sus hijos saberes y prácticas tradicionales campesinas.

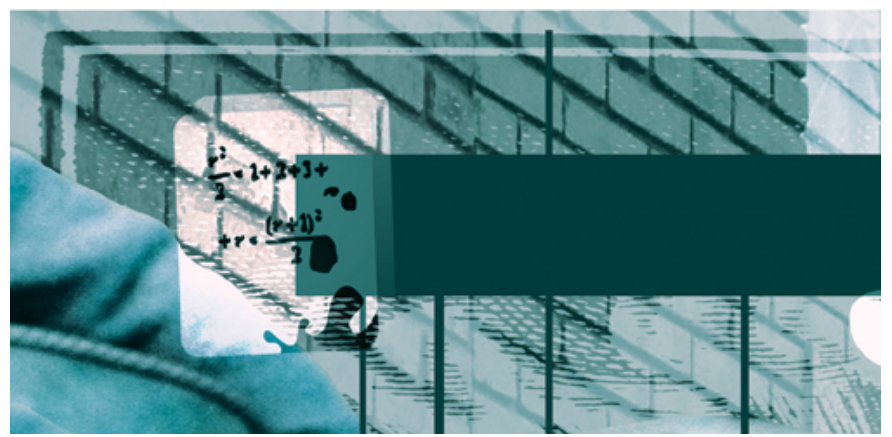




\section{Pesca artesanal}

Los pescadores tradicionales poseen saberes y prácticas que hacen parte de la historia de su región. La pesca artesanal en el Río Magdalena es una actividad que se ha desarrollado por siglos y ha tenido un impacto notable en la comunidad local.

Cuando hablamos de la Subienda en el Río Magdalena, hablamos de la pesca artesanal. Las personas que viven de la pesca tienen sus casas al lado del río, algunas comunidades diseñan sus casas de acuerdo al tiempo de creciente de los ríos. Esta es una mirada hacia lo tradicional, que posibilita el diálogo con lo científico. Uno en la clase podría hacer un paralelo entre la pesca industrial con la pesca artesanal (PCFI, No. 4, Licenciatura en educación básica con énfasis en ciencias naturales y educación ambiental, Universidad de Antioquia, entrevista colectiva).

Generar un diálogo entre el conocimiento científico y el tradicional, en la clase de ciencias, es posible desde el estudio de las características y diferencias entre la pesca artesanal e industrial, incluyendo su impacto social, económico y ambiental. Los saberes ancestrales en la pesca artesanal incluyen una interpretación ancestral de la dinámica de los ríos y representan una herencia generacional que hoy se está perdiendo por el abandono progresivo de esta tradición en las generaciones más jóvenes.

\section{Presencia de estudiantes pertenecientes a comunidades indígenas}

La migración de familias hace que hoy día sea una realidad la presencia de estudiantes pertenecientes a comunidades indígenas en la ciudad, ligada a hechos como el desplazamiento forzado, la desterritorialización, la falta de oportunidades en el campo y la pobreza extrema. En Valledupar es común observar en los colegios oficiales estudiantes de comunidades de arhuacos, wiwas, kogis y kankuamos; por ello, los profesores deben asumir nuevos retos en sus prácticas para tener en cuenta los saberes ancestrales.

En los colegios ahora vemos estudiantes indígenas, en la universidad deberían prepararnos para esto. Yo tengo en el salón dos estudiantes indígenas y a ellos se les dificulta mucho hablar y socializar sus ideas al resto del grupo, uno de ellos aún no habla bien el español. A mí me ha tocado aprender cosas de su comunidad y buscar otras estrategias para que ellos puedan aprender y compartir lo que saben, sus experiencias (PCFI, No. 4, Licenciatura en ciencias naturales y educación ambiental, Universidad Popular del César, entrevista colectiva).
Es importante incluir el desarrollo de competencias para la enseñanza de las ciencias en contextos de diversidad cultural en el plan de estudios de la licenciatura, con el fin de dar reconocimiento a la identidad y saberes de estudiantes afrodescendientes e indígenas; también es preciso conocer las políticas de inclusión de estudiantes indígenas en las instituciones educativas y revisar si el currículo plantea una enseñanza intercultural.

Ahora, frente a la inclusión de las comunidades, Campo (2018) presenta los resultados de una investigación acerca de los factores que inciden en la pérdida de identidad cultural de las etnias indígenas presentes en la institución educativa Alfonso López Pumarejo, en Valledupar, señalando especialmente agentes como la colonización, la desterritorialización y la globalización. Al tiempo, destaca la poca adaptación de estos estudiantes al enfrentar cosmovisiones y estilos de vida distintos; en la escuela se presentan enormes niveles de rechazo y burlas hacia la condición indígena, los estudiantes son tratados como si fueran personas atrasadas y los demás cuestionan sus creencias, incluso su vestuario (Campo, 2018).

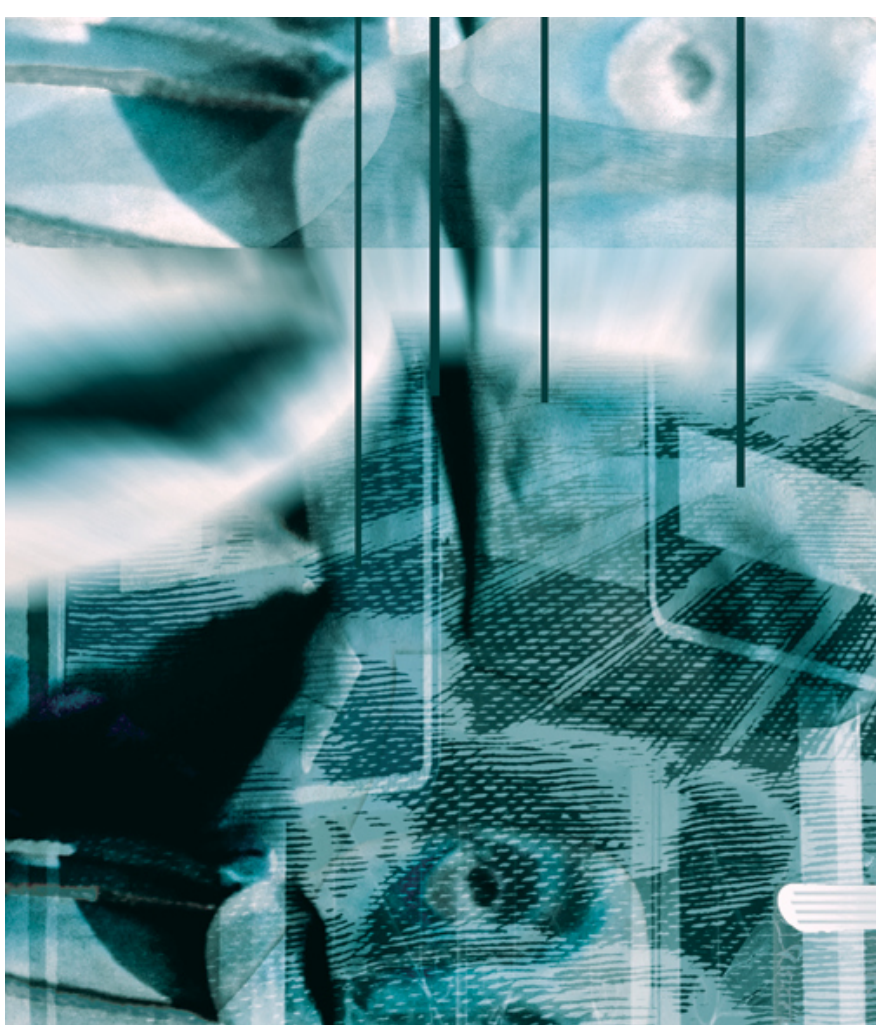




\section{Conclusiones}

Los profesores de ciencias en formación inicial plantean diferentes estrategias pedagógicas para integrar los saberes tradicionales y ancestrales a la enseñanza de las ciencias. Todos los profesores en formación entrevistados en la Licenciatura en Educación Básica con Énfasis en Ciencias Naturales y Educación Ambiental, de la Universidad del Cauca, manifestaron que vinculan los saberes ancestrales o tradicionales en su práctica pedagógica investigativa.

Los profesores en formación de los demás programas incluyen en menor proporción dichos saberes, siendo los docentes del programa de Licenciatura en Biología y Química de la Universidad de Caldas, y de la Licenciatura en Ciencias Naturales y Educación Ambiental, de la Universidad Popular del César, quienes vinculan este aspecto en menor grado. La relación entre el conocimiento científico y el saber ancestral/tradicional es un aspecto considerado también en algunos trabajos de grado.

Los profesores explicaron diferentes estrategias implementadas en la práctica pedagógica donde tienen en cuenta los saberes ancestrales y tradicionales y su relación con el conocimiento científico escolar. Sin embargo, manifiestan la necesidad de un cambio curricular orientado a la descolonización y el diálogo de saberes, que permita repensar la formación de profesores.

En cuanto al enfoque intercultural y la enseñanza de las ciencias, los profesores consideran vital reflexionar sobre la diversidad de saberes desde una perspectiva no eurocéntrica que permita trascender el cientificismo para recuperar el saber de comunidades indígenas, afrodescendientes y campesinas como formas válidas de conocer y producir conocimiento. Así, es fundamental que las instituciones educativas promuevan una enseñanza intercultural y contextualizada, que contribuya a visibilizar la naturaleza pluriétnica y multicultural del país; construir puentes interculturales desde la enseñanza de las ciencias permite trascender las fronteras culturales. En tal sentido, ésta requiere de profesores que identifiquen y establezcan los posibles cruces en las fronteras culturales del conocimiento, creando lazos entre las visiones de mundo estudiantil y las de la ciencia occidental (Aikenhead, 2002).

La inclusión de los saberes ancestrales, tradicionales y locales en la enseñanza de las ciencias busca rescatar nuestras raíces precoloniales. Las universidades públicas y los programas de licenciatura tienen una responsabilidad social y un compromiso por la reivindicación de los pueblos originarios, comunidades campesinas y afrodescendientes de todo el país. Ello implica considerar su derecho de conservar su lengua, saber y territorio, de acceder a una educación que garantice, no ya su mero reconocimiento, sino el resurgir de su cultura. En el ámbito pedagógico universitario se debe pasar, de un enfoque multicultural, a un enfoque intercultural contextualizado que permita la reafirmación de las culturas y el intercambio cultural, favoreciendo el acceso y la permanencia de jóvenes indígenas, afrodescendientes y campesinos en los programas de formación docente.

Además de incluir una perspectiva epistemológica estrictamente occidental, la formación inicial de profesores de ciencias requiere del estudio de las epistemologías del sur u otras afines. Así mismo, la historia y filosofía de las ciencias pueden tratarse desde una perspectiva intercultural en los programas de formación del profesorado; ello contribuirá a que el futuro profesor en ejercicio integre a su práctica pedagógica el diálogo de saberes, considerando distintas epistemes, cosmovisiones y prácticas.

En tal sentido, Santos (2009) destaca que las epistemologías del Sur realizan aportes valiosos al concepto de interculturalidad al verla como diálogo de saberes. Pensar en otra epistemología es hacerlo desde otras lógicas y racionalidades, en otras prácticas $\mathrm{y}$ formas de conocer que han sido negadas; implica construir el conocimiento desde lo inter epistémico, dando valor a diversos saberes y a sus respectivos discursos, analizando las asimetrías que históricamente se han evidenciado en grupos étnicos y culturales identitariamente diversos.

La ecología de saberes busca la complementariedad entre diferentes tipos de conocimientos sin que exista ningún tipo de jerarquía, con el fin de evitar la violencia epistémica y el epistimicidio de los saberes ancestrales (propios) o de conocimientos distintos al conocimiento científico debido al colonialismo europeo y estadounidense. 


\section{Referencias}

Aikenhead, G. (2002). Cross-Cultural science teaching: Rekindling traditions for aboriginal students. Canadian Journal of Science, Mathematics and Technology Education, 2(3), pp. 287-304. DOI: https:// doi.org/10.1080/14926150209556522

Aguado, T., y Malik, B. (2009). Intercultural education in higher education: Challenges and opportunities. Intercultural Education, 20(3), pp. 201-202. DOI: https://doi.org/10.1080/14675980903138426

Boen, P., Piñuel, L., Zubillaga, F., Dalzotto, D., y Sharry, S. (2017). Revalorizar el Saber Ancestral: Una experiencia de extensión con nuestros adultos mayores. Revista Facultad de Agronomía de la Universidad de la Plata. Número Especial, Extensión rural, pp. 87-96.

Cajigas, J. (2017). Ontoepistemologías indígenas. Tabula Rasa, No. 26, pp. 123-139.

Campo, O. M. (2018). Factores que inciden en la pérdida de identidad cultural de las etnias indígenas presentes en la Institución Educativa Alfonso López Pumarejo de Valledupar. Tesis de pregrado, Licenciatura en Etnoeducación, no publicada, Universidad Abierta y a Distancia, Valledupar.

Creswell, J. (2005). Educational research: Planning, conducting, and evaluating quantitative and qualitative research. Upper Saddle River: Pearson Education.

Fernández, M., Torres, E., y García, C. (2016). Creencias sobre la educación intercultural y prácticas de enseñanza de profesores de secundaria de la población indígena Yaqui. Perfiles educativos, V. 38, No. 152, pp. 109-127. DOI: https://doi.org/10.22201/iisue.24486167e.2016.152.57599

Francis, J. K., y Lowe. C. (2000). Bioecología de árboles nativos y exóticos de Puerto Rico y las Indias Occidentales. Washington: United States Department of Agriculture, pp. 148-151.

Funtowicz, S., y Ravetz, J. (1993). Science for the post-normal age. Futures, 25(7), pp. 739-755. DOI: https://doi.org/10.1016/0016-3287(93)90022-L

Gómez, G., Galindo, A., García, A., y González, L. (2014). Enseñanza de la evolución desde una perspectiva intercultural dialógica. Revista Citecsa, Vol. 5. No. 8, pp 66-78.

Leeman, Y., y Ledoux, G. (2003). Preparing teachers for intercultural education. Teaching Education, 14(3), pp. 279-291. DOI: https://doi.org/10.1080/1047621032000135186

Leiva, J. (2010). Práctica de la interculturalidad desde la perspectiva docente: Análisis y propuestas pedagógicas. Cultura y Educación, V. 22, pp. 67-8. DOI: https://doi.org/10.1174/113564010790935240

MEN. (1994). Ley General de Educación, Ley 115 de febrero 8 de 1994. Bogotá: MEN.

Ministerio de Educación Nacional (MEN). (2013). Sistema colombiano de formación de educadores $y$ lineamientos de política. Obtenido desde http://www.colombiaaprende.edu.co/html/productos/1685/ articles-338720_documento_final.pdf

Molina, A. (2017). Algunas aproximaciones a una perspectiva intercultural: Entre discursos generales de la educación y específicos centrados en la naturaleza de lo que se quiere enseñar. Tecné, Episteme y Didaxis, No. 42, pp. 7-21. 
Molina, A., y Mojica, L. (2013). Enseñanza como puente entre conocimientos científicos escolares y conocimientos ecológicos tradicionales. Magis. Revista Internacional de Investigación en Educación, 6(12), pp. 37-53.

Molina, A., Mosquera, C., Utges, G., Mojica, L., Cifuentes, C., Reyes, D., y Pedreros, R. (2014). Concepciones de los profesores sobre el fenómeno de la diversidad cultural y sus implicaciones en la enseñanza de las ciencias. Bogotá: Universidad Distrital, Doctorado Interinstitucional en Educación.

Molina, A., y Utges, G. (2011). Diversidad cultural, concepciones de los profesores y los ámbitos de sus prácticas. Dos estudios de caso. Revista de Enseñanza de la Física, 24(2), pp. 7-26.

Mortimer, E., y El Hani, C. (2014). Conceptual Profi les a theory of teaching and learning scientific concepts. Springer.

Ohshima, T., Tamura, T., y Sato, M. (2007). Influence of pulsed electric field on various enzyme activities. Journal of Electrostatics, No. 65, pp. 156-161. DOI: https://doi.org/10.1016/j.elstat.2006.07.005

Peña, C. M. (2014). Conocimiento escolar y saberes campesinos. Encuentros y desencuentros en la escuela rural. Praxis educativa, No. 15, pp. 103-123. DOI: https://doi.org/10.26620/uniminuto. praxis.14.15.2014.103-123

Rodríguez, E. (2002). Concepciones de práctica pedagógica. Revista de la facultad de artes y humanidades, Universidad Pedagógica Nacional, No. 16, pp. 1-34.

Sánchez, O. (2017). Educación propia, etnoeducación e interculturalidad. ¿Por qué no permitir una autonomía radical a quienes al menos no han acabado con el planeta? El Tiempo. Obtenido desde https://www.eltiempo.com/opinion/columnistas/oscar-sanchez/educacion-propia-etnoeducacione-interculturalidad-oscar-sanchez-86832

Santos, B. S. (2009). Una epistemología del sur: La reinvención del conocimiento y la emancipación social. México: Siglo XXI, CLACSO.

Santos, G. (2014). Do cientificismo ao diálogo intercultural na formação do professor e ensino de ciências. Interacções, No. 31, pp. 28-53.

Schmelkes, S. (2004). La educación intercultural: Un campo en proceso de consolidación. Revista Mexicana de Investigación Educativa, 9(20), pp. 9-13.

Sevilla, M., y Domingo, F. (2107). Guía (incompleta) al Festival de Música del Pacífico Petronio Álvarez, 19972016. Santiago de Cali: Pontificia Universidad Javeriana, Sello Editorial Javeriano, pp. 1-142.

Scarpetta, L. (2017). Reconocimiento fitoquímico y etnobotánico de Erythroxylum coca en la población Nasa del Departamento del Cauca, Colombia. Revistas Unilibre, 14(1), pp. 21-46.

Stake, R. (1999). Investigación con estudio de casos. Madrid: Morata, p. 52.

Yuen, C. Y. (2009). Dimensions of diversity: Challenges to secondary school teachers with implications for intercultural teacher education. Teaching and Teacher Education, pp. 1-10. 